\title{
The roles of ncRNAs in the diagnosis, prognosis and clinicopathological features of breast cancer: a systematic review and meta-analysis
}

\author{
Shihui Tang ${ }^{1}$, Wei Fan ${ }^{1,2}$, Jiang Xie ${ }^{3}$, Qiaoling Deng ${ }^{1}$, Ping Wang ${ }^{1}$, June Wang ${ }^{1}$, \\ Peipei $\mathbf{X} \mathbf{u}^{1}$, Zheng Zhang ${ }^{1}$, Yirong $\mathbf{L i}^{1}$ and Mingxia $\mathbf{Y u}^{1}$ \\ ${ }^{1}$ Department of Clinical Laboratory \& Center for Gene Diagnosis, Zhongnan Hospital of Wuhan University, Wuhan, Hubei, \\ 430071, China \\ ${ }^{2}$ Department of Pathology, Zhongnan Hospital of Wuhan University, Wuhan, Hubei, 430071, China \\ ${ }^{3}$ Department of Otorhinolaryngology and Head \& Neck Surgery, Hunan Children's Hospital, University of South China \\ Hengyang, Hunan, 421000, China \\ Correspondence to: Mingxia YU, email: dewrosy520@163.com \\ Yirong Li, email: YirongLi_Whu@163.com \\ Keywords: noncoding RNA, breast cancer, meta-analysis, prognosis, diagnosis \\ Received: November 07, $2016 \quad$ Accepted: April 12, $2017 \quad$ Published: August 10, 2017 \\ Copyright: Tang et al. This is an open-access article distributed under the terms of the Creative Commons Attribution License 3.0 (CC BY 3.0), \\ which permits unrestricted use, distribution, and reproduction in any medium, provided the original author and source are credited.
}

\section{ABSTRACT}

Background: A number of studies have shown that noncoding RNAs (ncRNAs) are abnormally expressed in breast cancers. However, the roles of ncRNAs remain unclear in breast cancer. Here, we aim to investigate the potential diagnostic and prognostic roles of ncRNAs in breast cancer.

Methods: Comprehensive literature search in Medline and Web of Science and a meta-analysis were performed to identify the association between ncRNAs and diagnosis, prognosis, and clinicopathological features of breast cancer.

Results: A total of 103 eligible studies, involving16, 828 independent participants, were included in the meta-analysis. In total, there were 98 individual and 11 grouped ncRNAs. 51 studies were eligible for survival analysis, 27 studies were eligible for diagnostic analysis, and 46 studies were eligible for clinicopathological features analysis. The abnormal expression of ncRNAs is associated with OS, RFS and PFS in breast cancer patients. For the diagnosis value of ncRNAs, the pooled OR and 95\% CI for sensitivity, specificity, DOR and AUC on all ncRNAs were 0.83 [95\% CI: $0.82-0.84$ ] 0.80 [95\% CI: 0.79- 0.82], 24.77 [95\% CI: 17.44- 35.16] and 0.9037, respectively. The analysis showed that downregulation of ncRNAs in breast cancer was associated with decreased risk of LNM, increased tumor size and PR expression, whereas, upregulation of ncRNAs was associated with increased HER2 expression.

Conclusions: High expression of ncRNAs was associated with poor OS, RFS, and PFS, while low expression of ncRNAs was related to favorable OS and RFS. Meanwhile, ncRNAs have potential diagnostic value for breast cancer.

\section{INTRODUCTION}

Breast cancer is the leading cause of cancer death in women. Early diagnosis and treatment are crucial to improve survival rate and quality of life of breast cancer patients [1]. However, because the primary breast cancers often lack typical clinical manifestations, many patients have been in advanced stage at the time of diagnosis [2].
Therefore, it is very important to find good breast cancer biological markers.

NcRNAs regulate cell differentiation, polarity, and epithelial to mesenchymal transition in breast cancer [3]. In recent years, it is known that ncRNAs served as prognosis factors in breast cancer [1, 4]. For example, $\mathrm{Yu}$ et al. found that down-regulation of miR-129-5p induced EMT in breast cancer cells and is associated 
with poor prognosis [5]. However, whether or not and how ncRNAs could be used in diagnosing and predicting prognosis of breast cancer patients remain to be determined. Systematic review and meta-analysis of data from individual studies can help to evaluate the potential clinical value of ncRNAs. This study aimed to assess the association between ncRNAs (miRNAs and long noncoding RNAs (lncRNAs)) and prognosis, diagnosis, and clinicopathological features of breast cancer.

\section{RESULTS}

\section{Description of studies}

The PRISMA flow chart (Figure 1) summaries the selected studies in the review. We followed the PRISMA writing specification [6]. Our search yielded a total of 8981 reports. This was reduced to 6283 after removal of 2698 duplicates. 6055 were excluded after screening the titles and abstracts. Full-text articles were obtained for 138 studies, of which 103 were eligible for meta-analysis. 51 studies included survival data, 27 studies included diagnostic data, and 46 studies included clinicopathological features which included age, lymph node metastasis, tumor size, ER, PR, HER2, and menopausal. Low expression of ncRNAs was found in 30 studies while high expressions of ncRNAs were found in 64 studies. 32 articles investigated lncRNA, and 71 articles studied microRNA.

Micro-21 is the most commonly studied ncRNA [7-17], and all of these studies investigated its diagnostic and prognostic values in breast cancer. However, other ncRNAs showed opposite role. Madhavan [18] deemed the upregulation of miR-200b and miR-22 as unfavorable prognostic factors, while both Yao [19] and Chen [20] demonstrated that these two had potential favorable effect on breast cancer. Besides, Lu [21] proposed that breast cancer patients with high expression of HOX transcript antisense RNA (HOTAIR) had lower risks of relapse and mortality than those with lower expression. On the contrary, Gupta [22] considered that HOTAIR could reprogram chromatin state to promote breast cancer metastasis and death.

\section{Prognosis}

51 studies reporting survival analysis were eligible for the meta-analyses (Supplementary Table 1). 44 studies reported overall survival (OS), 9 studies reported recurrence-free survival (RFS), and 4 studies reported progression/event/disease-free survival (PFS) (Table 1). Summarized hazard ratio (HR) was used as effect size to estimate the relationship between expression of ncRNAs and breast cancer survival. Random-effects model was used for the pooled analysis to detect heterogeneity. In the 44 studies reporting OS, high expression of 19 ncRNAs was associated with improved breast cancer survival rates $(\mathrm{HR}=0.33$, 95\% confidence interval $(95 \% \mathrm{CI}): 0.23$ 0.47) (Supplementary Figure 1B) and 21 ncRNAs were associated with an increased risk of death $(\mathrm{HR}=2.63,95 \%$ CI: 2.27-3.05) (Supplementary Figure 1A).

Seven ncRNAs were investigated in more than two studies: miR-21 ( $\mathrm{n}=8$ studies), miR-200a ( $\mathrm{n}=2$ studies), miR-200b ( $\mathrm{n}=2$ studies), miR-200c ( $\mathrm{n}=2$ studies), miR-22 ( $\mathrm{n}=2$ studies), miR-124 ( $\mathrm{n}=2$ studies), miR-210 ( $\mathrm{n}=2$ studies), and HOTAIR ( $\mathrm{n}=2$ studies). By combining these studies, the pooled HR and $95 \%$ CI were as follows: miR-21 $(\mathrm{HR}=1.97,95 \%$ CI: 1.55-2.44); miR-200a $(\mathrm{HR}=3.24$, 95\% CI: 1.30-8.07); miR-200b $(\mathrm{HR}=2.08$, 95\% CI: 0.54-8.01); miR-200c (HR=3.41, 95\% CI: $1.91-$ 6.09); miR-22 (HR=0.88, 95\% CI: 0.36-2.19); miR-124 $(\mathrm{HR}=0.71,95 \% \mathrm{CI}: 0.55-0.92) ; \mathrm{miR}-210(\mathrm{HR}=0.71,95 \%$ CI: $0.55-0.92)$; HOTAIR (HR=1.21, 95\% CI:0.16-8.93) (Supplementary Figure 3).

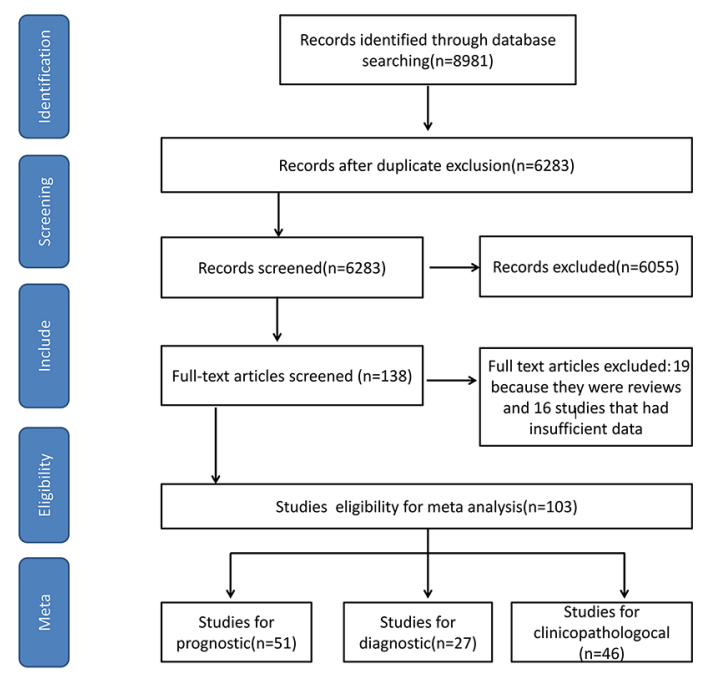

Figure 1: The PRISMA flow chart for selecting studies in Systematic Review and Meta-analysis. 
Table 1: Summary HR of ncRNAs for breast cancer

\begin{tabular}{lccccc}
\hline Survival analysis & & No. of studies & No. of patients & Pooled HR & Heterogeneity \\
\hline OS & Down & 19 & 4472 & $0.33[0.23-0.47]$ & $79 \%$ \\
& Up & 25 & 6854 & $2.63[2.27-3.05]$ & $53 \%$ \\
RFS & Down & 4 & 2605 & $0.68[0.53,0.87]$ & $67 \%$ \\
& Up & 5 & 7447 & $2.70[1.91,3.81]$ & $0 \%$ \\
PFS & Down & 1 & 57 & $0.40[0.17,0.94]$ & $/$ \\
& Up & 3 & 442 & $2.09[1.41,3.11]$ & $64 \%$ \\
\hline
\end{tabular}

Similarly, 9 studies were eligible for the RFS, with 4 down-regulated ncRNAs (HR $=0.71,95 \%$ CI: 0.55-0.92) and 5 up-regulated ncRNAs (HR $=2.70,95 \%$ CI: 1.91-3.81) were associated with risk of recurrence (Supplementary Figure 1D). After combining two studies, the result for MALAT1 showed a pooled HR of 2.36 (95\% CI: 1.55-3.60) in terms of RFS. For the PFS, three studies addressed 4 upregulated ncRNAs (HR=2.09, 95\% CI: 1.41-3.11) and one down regulated (Supplementary Figure 1C).

\section{Diagnosis}

Analysis of data from diagnostic accuracy studies including 27 original studies reported 1 lncRNA and 47 miRNAs (Supplementary Table 2). 13 ncRNAs are down regulated and the others are up regualted. All articles including 2287 patients and 1644 healthy control were published between 2010 and 2016. Significant heterogeneity was observed among the 27 studies in sensitivity and specificity analyses $\left(\mathrm{I}^{2}=86.1 \%\right.$ and $93.8 \%$, respectively). The pooled estimates for sensitivity, specificity, positive likelihood ratios (PLR), negative likelihood ratios (NLR), diagnostic odds ratios (DOR) and Area Under Curve (AUC) of all ncRNAs were 0.83 [95\% CI: 0.82-0.84], 0.80 [95\% CI: 0.79-0.82], 4.51 [95\% CI: 3.62-5.62], 0.21 [95\% CI: 0.17-0.25], 24.77 [95\% CI: 17.44-35.164] and 0.9037, respectively.

The most commonly studied ncRNA is miR-21 ( $\mathrm{n}=9$ studies). Pooled estimates of sensitivity, specificity, PLR, NLR, DOR and AUC associated with miR-21 were 0.77 [95\% CI: 0.74-0.82], 0.84 [95\% CI: 0.78- 0.88], 4.25 [95\% CI: 2.40- 7.52], 0.23 [95\% CI: 0.12- 0.46], 18.141 and 0.8982 (Supplementary Figure 4; Supplementary Figure 5; Supplementary Figure 6).

\section{Clinicopathological features}

46 studies including 24 microRNAs and 15 lncRNAs were included in analyses of the relationship between ncRNA and clinicopathological features (Supplementary Table 3), such as age, lymph node metastasis (LNM), tumor size, estrogen receptor (ER), progesterone receptor (PR), human epidermalgrowth factor receptor-2 (HER2), and menopausal factors (Table 2). 24 ncRNAs were up-regulated and 15 ncRNAs were down-regulated in breast cancer. The results suggested that upregulation of ncRNAs was positively correlated with the expression of HER2 (odd ratio (OR) $=1.36,95 \%$ CI: 1.10- 1.82). Meanwhile, an inverse correlation between downregulated ncRNAs and LNM (OR $=0.53,95 \%$ CI: 0.36 0.78 ), positive correlation between down-regulated ncRNAs and tumor size (OR $=1.47,95 \%$ CI: 1.19- 1.82) and the expression of PR (OR=1.33, 95\% CI: 1.05- 1.68) was noted (Figure 2). There were no other statistically significant associations between ncRNA and other factors (Supplementary Figure 7-Supplementary Figure 14).

\section{Subgroup analyses and meta-regression}

\section{Prognosis}

We performed meta-regression analysis but didn't found the publication year and RNA type which can explain the heterogeneity in up regulation ncRNAs $\left[\mathrm{Chi}^{2}=79.90, \mathrm{df}=36 \quad(\mathrm{P}<0.0001) ; \mathrm{I}^{2}=55 \%\right]$. Then, we performed subgroup analysis based on races and sample type. There was no significant heterogeneity in the tissue microarrays subgroup $\left[\mathrm{Chi}^{2}=2.60, \mathrm{df}=4(\mathrm{P}=0.63)\right.$; $\left.\mathrm{I}^{2}=0 \%\right]$ and blood subgroup $\left[\mathrm{Chi}^{2}=2.57, \mathrm{df}=7(\mathrm{P}=0.28)\right.$; $\left.\mathrm{I}^{2}=22 \%\right]$. But significant heterogeneity $\left[\mathrm{Chi}^{2}=62.74\right.$, $\left.\mathrm{df}=30(\mathrm{P}=0.0004) ; \mathrm{I}^{2}=52 \%\right]$ was observed in fresh tissue subgroup (Figure 3B).

\section{Diagnosis}

To study the diagnostic value, we performed subgroup analysis based on sample type. We found that the ncRNAs extracted from blood have higher sensitivity, specificity and AUC than those from tissue (Figure 4). Furthermore, diagnosis based on multiple ncRNAs showed higher accuracy than signle ncRNA (Table 3, and Figure 5). This information revealed a high potential diagnostic value of multiple ncRNAs from blood for breast cancer detection.

\section{Influence analysis and publication bias}

In influence analysis for studies on highly expressed ncRNA, pooled results were not substantially altered 
Table 2: Summary diagnostic accuracy of ncRNAs for breast cancer

\begin{tabular}{lcccccc}
\hline Analysis & No. & Pooled Sen & Pooled Spe & PLR & NLR & AUC \\
\hline Sum & 27 & $0.83(0.82-0.84)$ & $0.80(0.79-0.82)$ & $4.51(3.62-5.62)$ & $0.21(0.17-0.25)$ & 0.9037 \\
ncRNA profiles & & & & & & \\
combine & 7 & $0.86(0.84-0.89)$ & $0.87(0.84-0.89)$ & $7.22(4.26-12.23)$ & $0.16(0.11-0.24)$ & 0.9240 \\
single & 24 & $0.82(0.80-0.83)$ & $0.79(0.77-0.80)$ & $4.16(3.28-5.26)$ & $0.22(0.18-0.27)$ & 0.8951 \\
Sample types & & & & & & \\
tissue & 7 & $0.81(0.79-0.83)$ & $0.77(0.74-0.80)$ & $3.75(2.48-5.67)$ & $0.26(0.18-0.39)$ & 0.8744 \\
blood & 22 & $0.83(0.82-0.84)$ & $0.82(0.80-0.84)$ & $4.93(3.838-6.34)$ & $0.18(0.15-0.23)$ & 0.9147 \\
\hline
\end{tabular}

Sen: sensitivity, Spe: specificity, PLR: Positive likelihood ratio, NLR: negative likelihood ratio, AUC: area under concentration-time curve

after removing any one of the studies, suggesting that the pooled result is stable. In contrast, in down regulated part, we found an outlier and once this outlier was excluded [23], $\mathrm{I}^{2}$ went down from $79 \%$ to $0 \%\left[\mathrm{Chi}^{2}=13.21, \mathrm{df}=17\right.$ $\left.(\mathrm{P}=0.72) ; \mathrm{I}^{2}=0 \%\right]$ (Figure 6B).

Risk of publication bias is a significant concern in prognostic studies [24]. Begg's funnel plot and Egger's test were used to evaluate the publication bias in the published literature [25]. For OS, Begg's funnel plot $(\mathrm{P}=0.045)$ and Egger's test $(\mathrm{P}=0.053)$ showed certain publication bias (Supplementary Figure 1; Supplementary Figure 2A). By using trim-and-fill Method, a symmetrical funnel plot was produced.
Hypothetical negative unpublished studies imputed to mirror the positive studies that cause funnel plot asymmetry (Figure 3B) [26]. The pooled analysis showed a negative relationship between high expression ncRNA and OS (HR, 0.82 [95\% CI, 0.65-0.99]), and the result has good stability (Supplementary Figure 1B). For low expression ncRNA, the result of Begg's funnel plot is opposite to Egger's test. After excluded the outlier as mentioned above, funnel plot transformed to symmetry.

The Deek's funnel plot asymmetry test was used to evaluate the publication bias in diagnostic analysis [25]. The result $(\mathrm{P}=0.12)$ confirmed that there was no significant publication bias (Supplementary Figure 6).

\section{A}

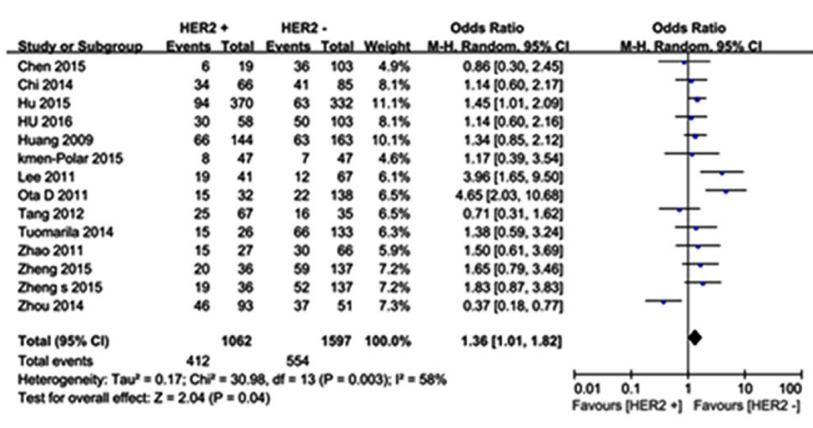

C

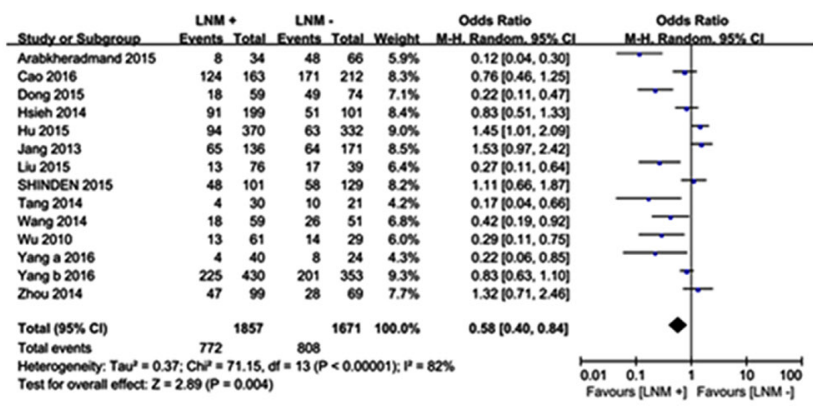

B

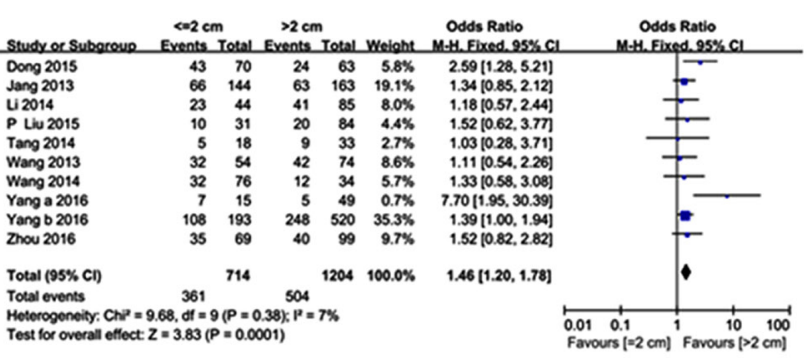

D

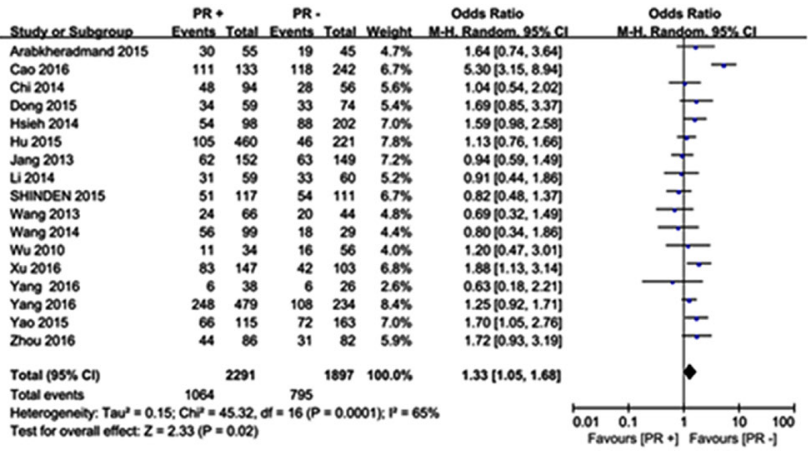

Figure 2: Forest plots for Clinicopathological features. Up-regulated ncRNAs : (A) Her2; down-regulated ncRNAs: (B) Tumor size; (C) Lymph node metastasis; (D) PR. 
Influence analysis and publication bias examination were implemented for every clinicopathological feature, in highly and lowly expressed ncRNAs, respectively.

A

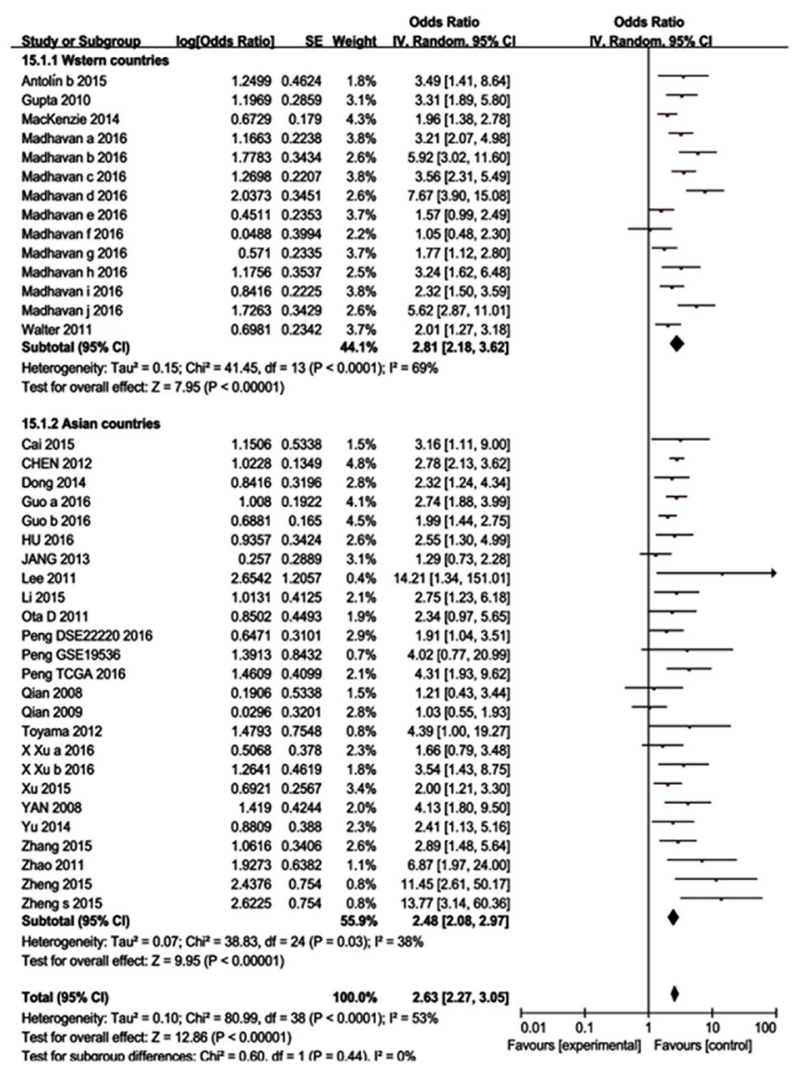

P-values from Begg's funnel plot and Egger's test were all larger than 0.05 . Since the studies of down-regulated ncRNAs were less than twenty, we performed the Begg's
B

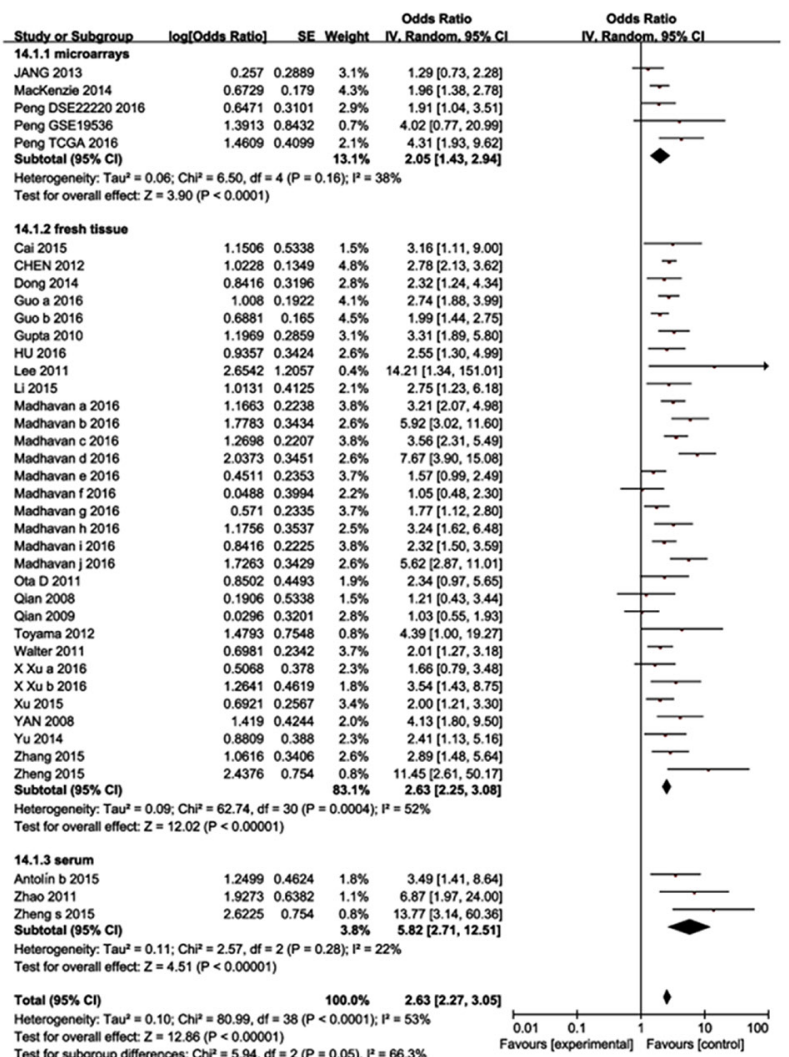

Figure 3: Subgroup analyses for OS of breast cancer patients. (A) Subgroup analyses based on races for up-regulated ncRNAs; (B) Subgroup analyses based on sample types for up-regulated ncRNAs.

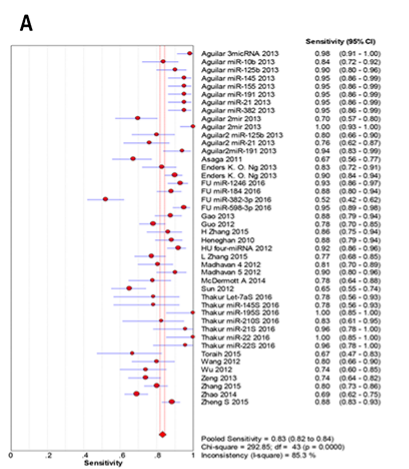

C

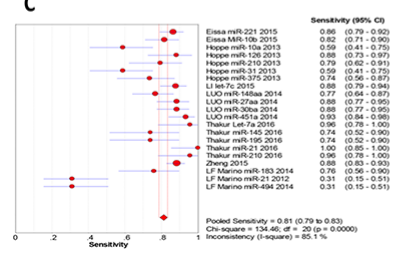

B

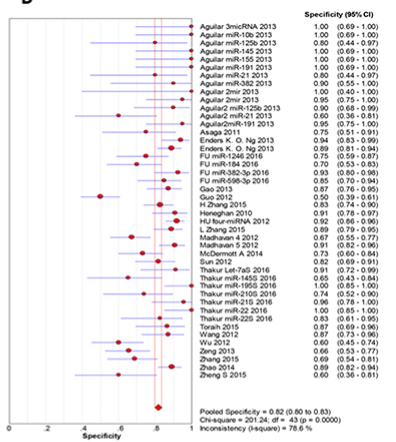

D

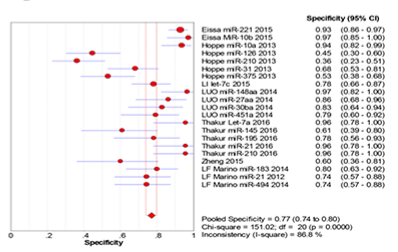

Figure 4: Sensitivity and specificity in subgroup analysis based on sample type. (A) the pooled sensitivity for blood; (B) the pooled specificity for blood; (C) the pooled sensitivity for tissues; (D) the pooled specificity for tissues. 
funnel plot and founded the funnel is asymmetric. Meanwhile, influence analysis results manifested the stability of results. In the high expression series, only HER2 group existed significant publication bias.

\section{DISCUSSION}

According to the GLOBOCAN estimates, about 14.1 million new cancer cases and 8.2 million deaths occurred in 2012 worldwide [27]. Human breast carcinoma is one of the most frequent cancers in the female and deeply threatens woman health and life quality. In this review, we gathered published papers to study the relationship between ncRNAs and prognosis, diagnosis, and clinicopathological features in breast cancer.

A

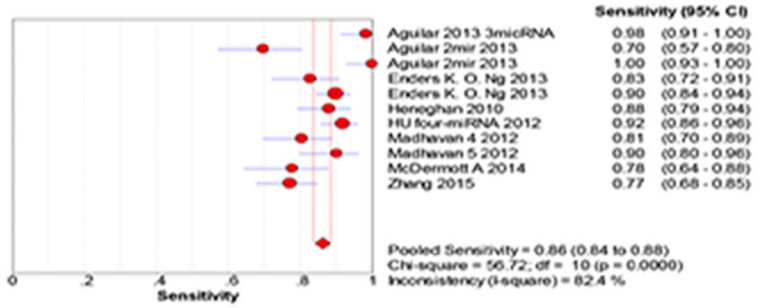

C

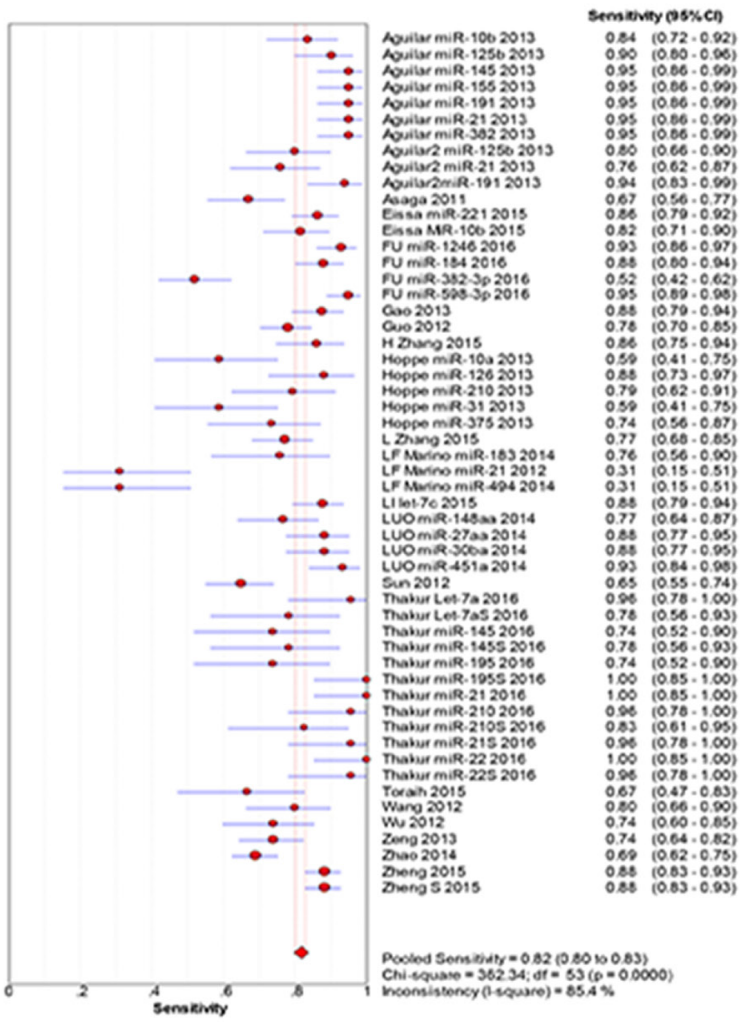

By systematically analyzing published original studies, we found that ncRNA have predictive value for prognosis in breast cancer. Combined HRs suggest that ncRNA are independent risk factors for OS in breast cancer patients. In addition, ncRNAs appear to be independent prognostic risk factors for RFS and PFS in breast cancer patients. However, these analyses had significant heterogeneity in terms of sample type and multivariable models. Large sample microarrays subgroup showed no significant heterogeneity but the effect size $(H R=1.83)$ was weaker than others, indicating the possibility of overestimating effect size. Besides, our finding also suggest that some ncRNAs alone (miR-124, miR-210, miR-21, miR-200a, miR-200c) are independent risk factors for OS in breast cancer.

\section{B}

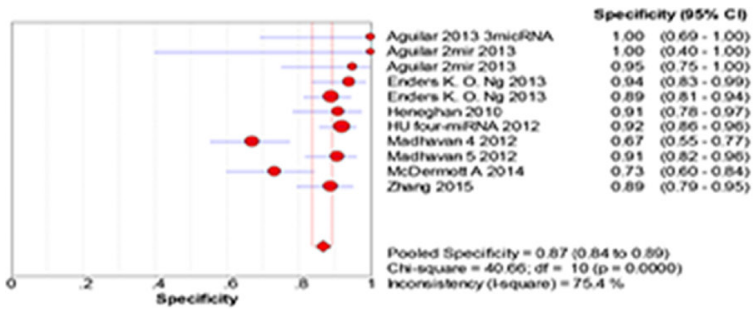

D

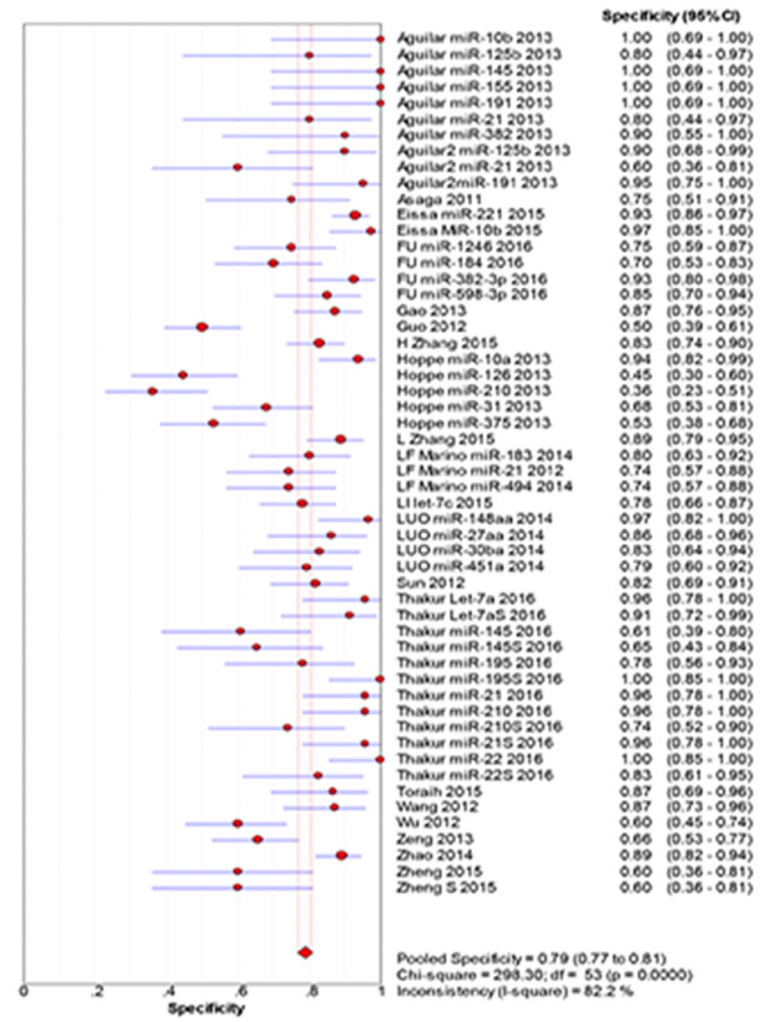

Figure 5: Sensitivity and specificity in subgroup analysis based on constituent. (A) the pooled sensitivity for single ncRNA; (B) the pooled specificity for single ncRNA; (C) the pooled sensitivity for multiple ncRNAs; (D) the pooled specificity for multiple ncRNAs. 
Table 3: Relationship between clinicopathological features and ncRNAs in breast cancer

\begin{tabular}{|c|c|c|c|c|c|}
\hline \multicolumn{2}{|c|}{ Survival analysis } & \multirow{2}{*}{$\begin{array}{c}\text { No. of studies } \\
10\end{array}$} & \multirow{2}{*}{$\begin{array}{c}\begin{array}{c}\text { No. of patients/ } \\
\text { controls }\end{array} \\
881 / 788\end{array}$} & \multirow{2}{*}{$\begin{array}{c}\text { Pooled HR } \\
1.05[0.80-1.38]\end{array}$} & \multirow{2}{*}{$\begin{array}{c}\text { Heterogeneity } \\
37 \%\end{array}$} \\
\hline Age & Down & & & & \\
\hline & Up & 15 & $934 / 1022$ & $1.01[0.84-1.22]$ & $0 \%$ \\
\hline \multirow[t]{2}{*}{ LNM } & Down & 13 & $1721 / 1500$ & $0.58[0.40,0.84]$ & $81 \%$ \\
\hline & $\mathrm{Up}$ & 17 & $1480 / 1441$ & $1.00[0.73,1.36]$ & $70 \%$ \\
\hline \multirow[t]{2}{*}{ Tumor Size } & Down & 9 & $714 / 1204$ & $1.47[1.19,1.82]$ & $7 \%$ \\
\hline & Up & 18 & $1265 / 1596$ & $0.80[0.60,1.05]$ & $65 \%$ \\
\hline \multirow[t]{2}{*}{ ER } & Down & 15 & $2499 / 1535$ & $1.25[0.98,1.61]$ & $66 \%$ \\
\hline & $\mathrm{Up}$ & 25 & $2133 / 1818$ & $0.91[0.73,1.14]$ & $59 \%$ \\
\hline \multirow[t]{2}{*}{ PR } & Down & 16 & 2291/1897 & $1.33[1.05,1.68]$ & $65 \%$ \\
\hline & Up & 23 & $1964 / 1981$ & $1.15[0.94,1.41]$ & $50 \%$ \\
\hline \multirow[t]{2}{*}{ HER2 } & Down & 11 & $849 / 1389$ & $0.68[0.42,1.11]$ & $85 \%$ \\
\hline & Up & 14 & $1062 / 1597$ & $1.36[1.10,1.82]$ & $58 \%$ \\
\hline \multirow[t]{2}{*}{ Menopausal } & Down & 2 & $166 / 159$ & $1.11[0.72,1.69]$ & $0 \%$ \\
\hline & Up & 11 & $866 / 917$ & $1.13[0.93,1.36]$ & $15 \%$ \\
\hline
\end{tabular}

The detection methods for breast cancer such as imaging examination and pathological examination had certain limitations, including radiation, invasion and low diagnostic accuracy. Noninvasive biomarkers, such as CEA and CA15-3, are widely used in clinic. However, these markers have low sensitivity and specificity for breast screening [9, 28]. Zeng et al [29] have demonstrated that these markers may be more suitable for advanced breast cancer. Thus, it is important to identify effective and noninvasive tumor biomarkers for early detection and diagnosis. In this meta-analysis, the overall AUC of SROC is 0.9037 , indicating a high accuracy of ncRNAs. The DOR value also evaluates the accuracy of a diagnostic test, with higher values indicating better performance[30]. DOR value less than 1.0 does not approve its competent role as a biomarker in the diagnostic test [31]. Nevertheless, the pooled DOR in this work reached up to 24.767 , suggesting ncRNA can be used as a non-invasive indicator for breast
A

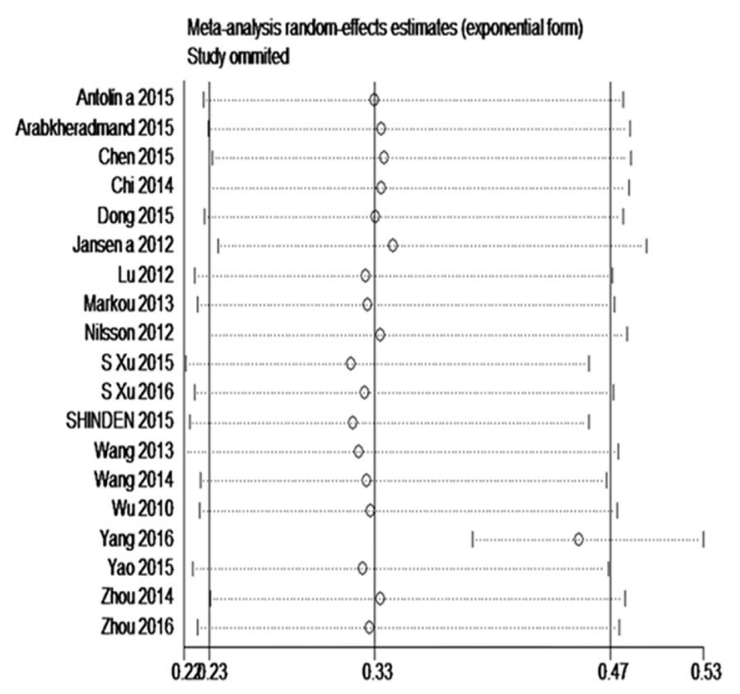

B

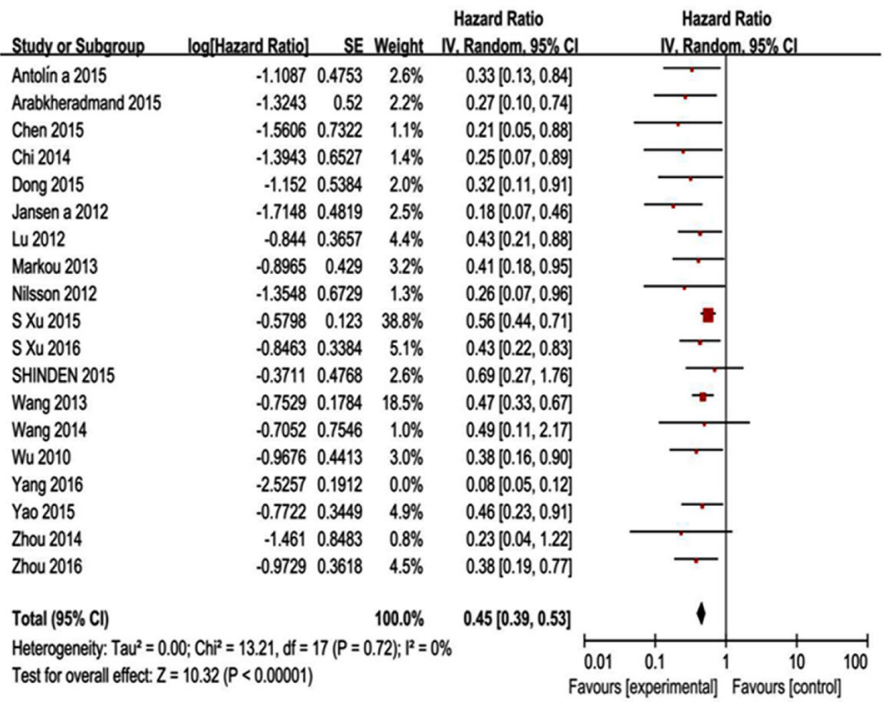

Figure 6: Subgroup analyses for OS of breast cancer patients. (A) Influence analysis for down-regulated ncRNAs; (B) Forest plots for down-regulated ncRNAs after excluding the outlier. 
cancer diagnosis. Besides, the sensitivity and specificity of multiple ncRNAs [18, 32-37] are higher than the single ones, suggesting combined ncRNAs could be useful for the diagnosis of breast cancer.

The threshold effect is one of the leading causes of heterogeneity in a diagnostic meta-analysis [38], and mainly caused by the different cut-off values used in individual studies [39]. Heterogeneity generated by non-threshold effect is usually addressed via pooled DOR [30]. The P values in Cochran's Q test were all less than 0.01 in our study, accompanied by $\mathrm{I}^{2}$ more than $50 \%$, suggesting that heterogeneity in the overall and subgroup analyses was attributed to threshold effect and non- threshold effect. Deek's funnel plot asymmetry test indicated no significant publication bias in the diagnostic analysis.

In the clinicopathological features part, downregulated ncRNAs were negatively related to tumor size and LNM, but positively related to the expression of PR; and up-regulated ncRNAs were positively related to the expression of HER2. However, the result of the relationship between down-regulated ncRNAs and HER2 exist significant heterogeneity, and after excluding the article [19] that stood out in the influence analysis, heterogeneity no longer has significance (Supplementary Figure 10). The LNM group showed large heterogeneity as well, and subgroup analyses by ncRNA type and sample type failed to locate the source of heterogeneity. Influence analyses for other aspects showed fine stability.

Three ncRNAs (miR-2 $(n=6)$, MALAT $1(n=4)$, miR-124 ( $=2)$ ) were studied in more than two studies. Our comprehensively analyzed data suggested that the expression of miR-21 was positively related to HER2 $(\mathrm{OR}=2.74,95 \% \mathrm{CI}: 1.13-6.62)$, negatively related to ER $(\mathrm{OR}=0.57$, 95\% CI: 0.34-0.96) and positively related to age $(\mathrm{OR}=0.57,95 \% \mathrm{CI}: 0.35-0.95)$. Meanwhile, the expression of MALAT1 was negatively related to tumor size $(\mathrm{OR}=1.29,95 \% \mathrm{CI}: 1.05-1.59)$. The expression of mir-124 was negatively related to $\mathrm{LNM}(\mathrm{OR}=0.17,95 \%$ CI: 0.10-0.31).

Most of the meta-analysis only evaluated the relationship between a type of ncRNA and many cancers. In our study, data were pooled rationally. We have analyzed the role of all ncRNAs in breast cancer including prognosis, diagnosis, and clinicopathological features. Besides, single ncRNA was also estimated. In diagnosis, we analyzed single ncRNA and multiple ncRNAs, respectively. The results suggested joint detection is better than single detection.

\section{Limitations}

Most of the included ncRNAs have been studied only once previously, making it difficult to systematically evaluate the clinical value of one specific ncRNA. There are several other limitations in our study. First, this review is only based on the results of databases of published studies, and did not include study registers or gray literature. This could be the source of publication bias. Second, our meta-analysis combines multiple studies, which contains different types, samples, cutoff values, and HRs, all of which may cause statistical heterogeneity. Third, many potential biomarkers being analyzed in our study are not used in clinical practice yet.

\section{CONCLUSION}

Taken together, our results indicated the potential value of ncRNAs in the prognosis and diagnosis of breast cancer. But considerable shortcoming might influence our final estimates. Therefore, future standardized researches with high quality are needed to verify these results.

\section{MATERIALS AND METHODS}

\section{Study strategy and eligibility criteria}

We conducted comprehensive literature searches in Medline and Web of Science for eligible studies up to Sep, 2016, using the keywords[(ncRNAs or microRNAs or miRNAs or long non-coding RNAs) AND (breast or breast cancer)], with publication language limited to English.

Case-control reports were identified that explored the association of any single or combination of relevant ncRNAs with one or more of the following aspects: survival, diagnosis and clinical features of breast cancer patients.

In order to be eligible for inclusion in meta-analysis, studies had to provide the effect size and CI for the association of ncRNAs with outcomes, or appropriate data for the effect size and CI could be calculated. For survival analysis, we extracted the HRs and 95\% CI [40] for overall survival (OS, duration of time from day of diagnosis to the day of death due to any cause), recurrence-free survival (RFS, duration of time from day of cure from cancer to the day evidence of cancer progression/recurrence is identified), progression/event/disease-free survival (PFS, duration of time from the day of first treatment to the day evidence of cancer progression are identified or the patient dies of any cause). In diagnostic articles, the sensitivity and specificity are extracted to construct twoby-two tables. ORs and 95\% CI were used to investigate the relationship between expression levels of ncRNAs and clinicopathological features. At the same time, we excluded review, letters, case reports, guidelines, and some studies without complete data.

Two reviewers independently screened titles and abstracts of all identified records according to prespecified inclusion and exclusion criteria. Disagreements were resolved by a third reviewer. Full text articles were obtained for all included studies and were screened again 
for inclusion or exclusion by two reviewers independently, with disagreements resolved by discussion.

\section{Quality assessment}

Three researchers independently reviewed and evaluated eligible studies assessed by the NewcastleOttawa quality assessment scale (NOS)[41, 42]. The methodological quality of diagnosis part in this study was performed with Quality Assessment of Diagnostic Accuracy Studies (QUADAS-2) criteria [41].

\section{Data extraction}

Data were extracted from eligible articles as follow. General information: first author's name, year of publication, country of the study, type of ncRNAs, sample size; characteristics of participants: (1) HRs and $95 \%$ CI were extracted for survival effect size. Since effect estimates extracted from multivariate analysis (e.g. Cox regression) that are affected by other variables, HR and 95\% CI were not directly extracted [10, 43-46]; approximations of HRs were indirectly calculated based on the correlative statistics using the methods described by Tierney et al [28]. When ncRNA expression levels were subdivided into low versus medium versus high groups, only data for the comparison between high versus low expression levels were extracted [14, 21, 47, 48]; (2) For diagnostic studies data were extracted relating to: sensitivity and specificity (two-by-two tables); (3) clinicopathological characteristics are age, tumor size, menopausal, lymph node metastasis, and the expression of growth factor receptors (estrogen receptor, ER; progesterone receptor, PR; epidermal growth factor receptor 2, HER2).

\section{Statistical analysis}

Data syntheses were conducted using Review Manager 5.2 and Meta-Disc 1.4. Publication bias examination, and influence analyses were performed using STATA 11.0. The HR with the corresponding 95\% CI for OS, RFS and PFS were calculated to evaluate the prognostic value of ncRNA $[49,50]$. HR $>1$ imply that patients with lower expression of the ncRNA had better prognosis than patients with higher expression. The true positive (TP), false positive (FP), false negative (FN), true negative (TN) based on two-by-two tables, that were used to consider sensitivity (SE), specificity (SP), positive likelihood ratio (PLR), negative likelihood ratio (NLR), and summary receiver operator characteristic (SROC) curve. These data were used to assess the diagnosis value of ncRNAs in breast cancer. Odds ratios (ORs) and the $95 \%$ CI were calculated to analyze the relationship between ncRNA and clinicopathological features. $\mathrm{P}<0.05$ was considered statistically significant. Heterogeneity was analyzed using the $\mathrm{Q}$ and $\mathrm{I}^{2}$ statistics $[41,42] . \mathrm{P}<0.1$ indicated presence of heterogeneity. $\mathrm{I}^{2}>50 \%$ was defined as significant heterogeneity.

\section{ACKNOWLEDGMENTS}

This study was supported by National Natural Science Funds (No. 81472033 and No. 30901308), the National Science Foundation of Hubei Province (No. 2013CFB233, No. 2013CFB235 and No.2016CFB672), the Scientific and technological project of Wuhan City (No. 2014060101010045), Hubei Province health and family planning scientific research project (WJ2015Q021), and Training Program of the science and technology innovation from Zhongnan Hospital of Wuhan University (cxpy20160054).

\section{CONFLICTS OF INTEREST}

All authors declare that there is no conflicts of interests.

\section{REFERENCES}

1. Matamala N, Vargas MT, Gonzalez-Campora R, Minambres R, Arias JI, Menendez P, Andres-Leon E, Gomez-Lopez G, Yanowsky K, Calvete-Candenas J, Inglada-Perez L, Martinez-Delgado B, Benitez J. Tumor microRNA expression profiling identifies circulating microRNAs for early breast cancer detection. Clin Chem. 2015; 61: 1098106. doi: 10.1373/clinchem.2015.238691.

2. Chen SJ, Chang $\mathrm{CH}$, Chen $\mathrm{KC}$, Liu CY. Association between depressive disorders and risk of breast cancer recurrence after curative surgery. Medicine (Baltimore). 2016; 95: e4547. doi: 10.1097/md.0000000000004547.

3. Sandhu GK, Milevskiy MJ, Wilson W, Shewan AM, Brown MA. Non-coding RNAs in Mammary Gland Development and Disease. Adv Exp Med Biol. 2016; 886: 121-53. doi: 10.1007/978-94-017-7417-8_7.

4. Malek E, Jagannathan S, Driscoll JJ. Correlation of long non-coding RNA expression with metastasis, drug resistance and clinical outcome in cancer. Oncotarget. 2014; 5: 8027-38. doi: 10.18632/oncotarget.2469.

5. Yu Y, Zhao Y, Sun XH, Ge J, Zhang B, Wang X, Cao XC. Down-regulation of miR-129-5p via the Twist1-Snail feedback loop stimulates the epithelial-mesenchymal transition and is associated with poor prognosis in breast cancer. Oncotarget. 2015; 6: 34423-36. doi: 10.18632/ oncotarget.5406.

6. Moher D, Liberati A, Tetzlaff J, Altman DG, Group P. Preferred reporting items for systematic reviews and 
meta-analyses: the PRISMA statement. BMJ. 2009; 339: b2535. doi: 10.1136/bmj.b2535.

7. Asaga S, Kuo C, Nguyen T, Terpenning M, Giuliano AE, Hoon DS. Direct serum assay for microRNA-21 concentrations in early and advanced breast cancer. Clin Chem. 2011; 57: 84-91. doi: 10.1373/clinchem.2010.151845.

8. Dong G, Liang X, Wang D, Gao H, Wang L, Wang L, Liu J, Du Z. High expression of miR-21 in triple-negative breast cancers was correlated with a poor prognosis and promoted tumor cell in vitro proliferation. Med Oncol. 2014; 31: 57. doi: 10.1007/s12032-014-0057-x.

9. Gao J, Zhang Q, Xu J, Guo L, Li X. Clinical significance of serum miR-21 in breast cancer compared with CA153 and CEA. Chin J Cancer Res. 2013; 25: 743-8. doi: 10.3978/j. issn.1000-9604.2013.12.04.

10. Huang GL, Zhang XH, Guo GL, Huang KT, Yang KY, Shen X, You J, Hu XQ. Clinical significance of miR-21 expression in breast cancer: SYBR-Green I-based real-time RT-PCR study of invasive ductal carcinoma. Oncol Rep. 2009; 21: 673-9.

11. Lee JA, Lee HY, Lee ES, Kim I, Bae JW. Prognostic Implications of MicroRNA-21 Overexpression in Invasive Ductal Carcinomas of the Breast. J Breast Cancer. 2011; 14: 269-75. doi: 10.4048/jbc.2011.14.4.269.

12. MacKenzie TA, Schwartz GN, Calderone HM, Graveel CR, Winn ME, Hostetter G, Wells WA, Sempere LF. Stromal expression of miR-21 identifies high-risk group in triplenegative breast cancer. Am J Pathol. 2014; 184: 3217-25. doi: 10.1016/j.ajpath.2014.08.020.

13. Mar-Aguilar F, Luna-Aguirre CM, Moreno-Rocha JC, Araiza-Chavez J, Trevino V, Rodriguez-Padilla C, ResendezPerez D. Differential expression of miR-21, miR-125b and miR-191 in breast cancer tissue. Asia Pac J Clin Oncol. 2013; 9: 53-9. doi: 10.1111/j.1743-7563.2012.01548.x.

14. Qian B, Katsaros D, Lu L, Preti M, Durando A, Arisio $\mathrm{R}, \mathrm{Mu} \mathrm{L}, \mathrm{Yu} \mathrm{H}$. High miR-21 expression in breast cancer associated with poor disease-free survival in early stage disease and high TGF-beta1. Breast Cancer Res Treat. 2009; 117: 131-40. doi: 10.1007/s10549-008-0219-7.

15. Toraih EA, Mohammed EA, Farrag S, Ramsis N, Hosny S. Pilot Study of Serum MicroRNA-21 as a Diagnostic and Prognostic Biomarker in Egyptian Breast Cancer Patients. Mol Diagn Ther. 2015; 19: 179-90. doi: 10.1007/ s40291-015-0143-6.

16. Walter BA, Gomez-Macias G, Valera VA, Sobel M, Merino MJ. miR-21 Expression in Pregnancy-Associated Breast Cancer: A Possible Marker of Poor Prognosis. J Cancer. 2011; 2: 67-75.

17. Yan LX, Huang XF, Shao Q, Huang MY, Deng L, Wu QL, Zeng YX, Shao JY. MicroRNA miR-21 overexpression in human breast cancer is associated with advanced clinical stage, lymph node metastasis and patient poor prognosis. Rna. 2008; 14: 2348-60. doi: 10.1261/rna.1034808.
18. Madhavan D, Peng C, Wallwiener M, Zucknick M, Nees J, Schott S, Rudolph A, Riethdorf S, Trumpp A, Pantel K, Sohn C, Chang-Claude J, Schneeweiss A, et al. Circulating miRNAs with prognostic value in metastatic breast cancer and for early detection of metastasis. Carcinogenesis. 2016; 37: 461-70. doi: 10.1093/carcin/bgw008.

19. Yao Y, Hu J, Shen Z, Yao R, Liu S, Li Y, Cong H, Wang X, Qiu W, Yue L. MiR-200b expression in breast cancer: a prognostic marker and act on cell proliferation and apoptosis by targeting Sp1. J Cell Mol Med. 2015; 19: 7609. doi: $10.1111 / \mathrm{jcmm} .12432$.

20. Chen B, Tang H, Liu X, Liu P, Yang L, Xie X, Ye F, Song C, Xie X, Wei W. miR-22 as a prognostic factor targets glucose transporter protein type 1 in breast cancer. Cancer Lett. 2015; 356: 410-7. doi: 10.1016/j.canlet.2014.09.028.

21. Lu L, Zhu G, Zhang C, Deng Q, Katsaros D, Mayne ST, Risch HA, Mu L, Canuto EM, Gregori G, Benedetto C, $\mathrm{Yu} \mathrm{H}$. Association of large noncoding RNA HOTAIR expression and its downstream intergenic $\mathrm{CpG}$ island methylation with survival in breast cancer. Breast Cancer Res Treat. 2012; 136: 875-83. doi: 10.1007/ s10549-012-2314-z.

22. Gupta RA, Shah N, Wang KC, Kim J, Horlings HM, Wong DJ, Tsai MC, Hung T, Argani P, Rinn JL, Wang Y, Brzoska P, Kong B, et al. Long non-coding RNA HOTAIR reprograms chromatin state to promote cancer metastasis. Nature. 2010; 464: 1071-6. doi: 10.1038/nature08975.

23. Yang F, Liu YH, Dong SY, Ma RM, Bhandari A, Zhang XH, Wang OC. A novel long non-coding RNA FGF14-AS2 is correlated with progression and prognosis in breast cancer. Biochem Biophys Res Commun. 2016; 470: 479-83. doi: 10.1016/j.bbrc.2016.01.147.

24. Altman DG. Systematic reviews of evaluations of prognostic variables. Bmj. 2001; 323: 224-8. doi:

25. Shan W, Sun C, Zhou B, Guo E, Lu H, Xia M, Li K, Weng D, Lin X, Meng L, Ma D, Chen G. Role of Dicer as a prognostic predictor for survival in cancer patients: a systematic review with a meta-analysis. Oncotarget. 2016; 7: 72672-72684. doi: 10.18632/oncotarget.12183.

26. Watzlawick R, Sena ES, Dirnagl U, Brommer B, Kopp MA, Macleod MR, Howells DW, Schwab JM. Effect and reporting bias of RhoA/ROCK-blockade intervention on locomotor recovery after spinal cord injury: a systematic review and meta-analysis. JAMA Neurol. 2014; 71: 91-9. doi: 10.1001/jamaneurol.2013.4684.

27. Torre LA, Bray F, Siegel RL, Ferlay J, Lortet-Tieulent J, Jemal A. Global cancer statistics, 2012. CA Cancer J Clin. 2015; 65: 87-108. doi: 10.3322/caac.21262.

28. Guo LJ, Zhang QY. Decreased serum miR-181a is a potential new tool for breast cancer screening. Int J Mol Med. 2012; 30: 680-6. doi: 10.3892/ijmm.2012.1021.

29. Zeng RC, Zhang W, Yan XQ, Ye ZQ, Chen ED, Huang DP, Zhang XH, Huang GL. Down-regulation of miRNA-30a 
in human plasma is a novel marker for breast cancer. Med Oncol. 2013; 30: 477. doi: 10.1007/s12032-013-0477-z.

30. Glas AS, Lijmer JG, Prins MH, Bonsel GJ, Bossuyt PM. The diagnostic odds ratio: a single indicator of test performance. J Clin Epidemiol. 2003; 56: 1129-35.

31. Liu L, Wang S, Cao X, Liu J. Analysis of circulating microRNA biomarkers for breast cancer detection: a metaanalysis. Tumour Biol. 2014; 35: 12245-53. doi: 10.1007/ s13277-014-2533-5.

32. Heneghan HM, Miller N, Kelly R, Newell J, Kerin MJ. Systemic miRNA-195 differentiates breast cancer from other malignancies and is a potential biomarker for detecting noninvasive and early stage disease. Oncologist. 2010; 15: 673-82. doi: 10.1634/theoncologist.2010-0103.

33. Hu Z, Dong J, Wang LE, Ma H, Liu J, Zhao Y, Tang J, Chen X, Dai J, Wei Q, Zhang C, Shen H. Serum microRNA profiling and breast cancer risk: the use of miR-484/191 as endogenous controls. Carcinogenesis. 2012; 33: 828-34. doi: 10.1093/carcin/bgs030.

34. McDermott AM, Miller N, Wall D, Martyn LM, Ball G, Sweeney KJ, Kerin MJ. Identification and validation of oncologic miRNA biomarkers for luminal A-like breast cancer. PLoS One. 2014; 9: e87032. doi: 10.1371/journal. pone.0087032.

35. Zhang H, Li B, Zhao H, Chang J. The expression and clinical significance of serum miR-205 for breast cancer and its role in detection of human cancers. Int J Clin Exp Med. 2015; 8: 3034-43.

36. Antolin S, Calvo L, Blanco-Calvo M, Santiago MP, Lorenzo-Patino MJ, Haz-Conde M, Santamarina I, Figueroa A, Anton-Aparicio LM, Valladares-Ayerbes M. Circulating miR-200c and miR-141 and outcomes in patients with breast cancer. BMC Cancer. 2015; 15: 297. doi: 10.1186/ s12885-015-1238-5.

37. Ng EK, Li R, Shin VY, Jin HC, Leung CP, Ma ES, Pang R, Chua D, Chu KM, Law WL, Law SY, Poon RT, Kwong A. Circulating microRNAs as specific biomarkers for breast cancer detection. PLoS One. 2013; 8: e53141. doi: 10.1371/ journal.pone.0053141.

38. Zamora J, Abraira V, Muriel A, Khan K, Coomarasamy A. Meta-DiSc: a software for meta-analysis of test accuracy data. BMC Med Res Methodol. 2006; 6: 31. doi: 10.1186/1471-2288-6-31.

39. Ji X, Guan C, Jiang X, Li H. Diagnostic accuracy of DNA methylation for head and neck cancer varies by sample type and number of markers tested. Oncotarget. 2016;7:8001980032. doi: 10.18632/oncotarget.12219.

40. Moser BK, McCann MH. Reformulating the hazard ratio to enhance communication with clinical investigators. Clin Trials. 2008; 5: 248-52. doi: 10.1177/1740774508091452.
41. Zeng X, Zhang Y, Kwong JS, Zhang C, Li S, Sun F, Niu $\mathrm{Y}, \mathrm{Du} \mathrm{L}$. The methodological quality assessment tools for preclinical and clinical studies, systematic review and metaanalysis, and clinical practice guideline: a systematic review. J Evid Based Med. 2015; 8: 2-10. doi: 10.1111/jebm.12141.

42. Stang A. Critical evaluation of the Newcastle-Ottawa scale for the assessment of the quality of nonrandomized studies in meta-analyses. Eur J Epidemiol. 2010; 25: 603-5. doi: 10.1007/s10654-010-9491-z.

43. Zheng R, Pan L, Gao J, Ye X, Chen L, Zhang X, Tang W, Zheng W. Prognostic value of miR-106b expression in breast cancer patients. J Surg Res. 2015; 195: 158-65. doi: 10.1016/j.jss.2014.12.035.

44. Li W, Jin X, Zhang Q, Zhang G, Deng X, Ma L. Decreased expression of miR-204 is associated with poor prognosis in patients with breast cancer. Int J Clin Exp Pathol. 2014; 7: 3287-92.

45. Tang H, Liu P, Yang L, Xie X, Ye F, Wu M, Liu X, Chen B, Zhang L, Xie X. miR-185 suppresses tumor proliferation by directly targeting E2F6 and DNMT1 and indirectly upregulating BRCA1 in triple-negative breast cancer. Mol Cancer Ther. 2014; 13: 3185-97. doi: 10.1158/1535-7163. mct-14-0243.

46. Tuomarila M, Luostari K, Soini Y, Kataja V, Kosma VM, Mannermaa A. Overexpression of microRNA-200c predicts poor outcome in patients with PR-negative breast cancer. PLoS One. 2014; 9: e109508. doi: 10.1371/journal. pone. 0109508 .

47. Toyama T, Kondo N, Endo Y, Sugiura H, Yoshimoto N, Iwasa M, Takahashi S, Fujii Y, Yamashita H. High expression of microRNA-210 is an independent factor indicating a poor prognosis in Japanese triple-negative breast cancer patients. Jpn J Clin Oncol. 2012; 42: 256-63. doi: 10.1093/jjco/hys001.

48. Jansen MP, Reijm EA, Sieuwerts AM, Ruigrok-Ritstier K, Look MP, Rodriguez-Gonzalez FG, Heine AA, Martens JW, Sleijfer S, Foekens JA, Berns EM. High miR-26a and low CDC2 levels associate with decreased EZH2 expression and with favorable outcome on tamoxifen in metastatic breast cancer. Breast Cancer Res Treat. 2012; 133: 937-47. doi: 10.1007/s10549-011-1877-4.

49. Peairs KS, Barone BB, Snyder CF, Yeh HC, Stein KB, Derr RL, Brancati FL, Wolff AC. Diabetes mellitus and breast cancer outcomes: a systematic review and meta-analysis. J Clin Oncol. 2011; 29: 40-6. doi: 10.1200/jco.2009.27.3011.

50. Wang Y, Zhang Y, Pan C, Ma F, Zhang S. Prediction of poor prognosis in breast cancer patients based on microRNA-21 expression: a meta-analysis. PLoS One. 2015; 10: e0118647. doi: 10.1371/journal.pone.0118647. 